\title{
LA FORMACIÓN DE PROFESORES EN FRANCIA
}

\author{
Por: ROLANDO CHABANNES
}

I. LA ESCUELA Y LA SOCIEDAD, CONSERVATISMO Y EVOLUCIONES

En su forma y modalidades institucionales actuales, el sistema francés de formación de profesores tiene menos de 15 años.

Las instituciones que tienen a su cargo lo esencial de esta formación fueron creadas entre 1980 y 1990, se trata de: Las Misiones Acádemicas para la Formación del Personal de la lo que se refiere a la formación continua y los Institutos Universitarios Educación Nacional (MAFPEN) en

de Formación de Maestros (IUFM) para lo que tiene que ver con la formación inicial.

Pero, digámoslo desde ahora, estas dos creaciones no acabaron totalmente con las instituciones existentes, por razones profundas llegadas a la tradición y la cultura nacional y me gustaría decir unas palabras sobre este aspecto, aunque la sociología y la historia de las instituciones no constituyen el tema central de mi intervención, porque ciertos aspectos del sistema que pueden aparecer extraños para los especialistas extranjeros, tienen sus rasgos particulares en la historia y en

la cultura francesas.

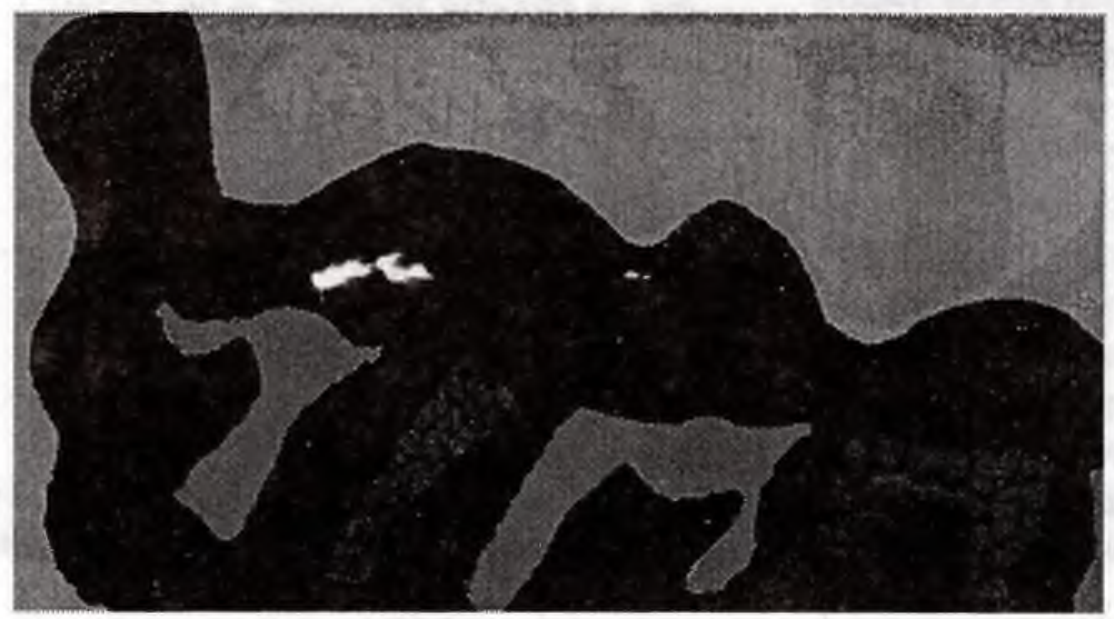

Nuestra cultura nacional se caracteriza por una extrema sensibilidad hacia todo aquello que se refiere con su escuela. La sociedad se reconocía simbólicamente 
en la escuela heredada de la Tercera República y en sus tres valores fundamentales: escuela pública, laica y obligatoria.

A manera de ejemplo, los asuntos educativos, son los únicos que han podido, en el transcurso de los últimos quince años, movilizar tanta gente como para hacer caer dos gobiernos. El actual presidente de la República había anunciado un referéndum sobre la escuela durante la campaña electoral; dicha proposición fuen bien acogida por un grupo electoral muy deseoso de pronunciarse sobre un tema tan cargado de símbolos; pero mirando un poco más de cerca, tratándose de grandes orientaciones educativas cualquier asunto puede generar una guerra escolar y cortar, como decimos, el país en dos. Parece más razonable orientarse hacia consultas parciales.

Es por ello que las innovaciones en este campo se hacen con prudencia, y aunque ha sido posible crear nuevas instituciones, ha sido mucho más difícil suprimir las antiguas debido a las prerrogativas que se les ha concedido.

Así, cuando en 1992 se renovó el Certificado de Aptitud para la Enseñanza Secundaria (CAPES), que es el principal concurso para la contratación de profesores de colegios y de liceos (Básica Secundaria y Media en Colombia ), introduciendo contenidos de carácter didáctico, no se introdujeron contenido semejantes en el otro concurso, el de la Agregación que no tuvo ninguna modificación. De la misma manera si los IUFM tienen la responsabilidad de la formación inicial de los maestros de primero y de segundo grado, la formación continua de los profesores, por el contrario, permanece bajo la responsabilidad de la administración departamental y regional.

$A$ veces se presentan situaciones complejas a nivel local, que podría citar ocasionalmente, pero sobre las cuales este preámbulo me evitará extenderme más ampliamente.

La configuración actual se sitúa, grosso modo, en la confluencia de dos corrientes.

G. Miallaret nos caricaturizaba mucho cuando en 1983 escribía: "Durante mucho tiempo se pensó que para ejercer la profesión docente bastaba tener un amplia cultura académica sin formación pedagógica..., o una buena formación pedagógica sin un nivel particular de formación académica...".

Era la antigua distinción entre la enseñanza secundaria y la enseñanza primaria. En efecto, la formación de las antiguas Escuelas Normales de Institutores, creadas en 1833 por la ley Guizot, desarrolladas y generalizadas en 1879 que preparaban para la enseñanza de primer grado se caracterizaba por una preocupación en torno a la formación profesional. La escuela normal estaba rodeada por una escuela anexa y escuela de aplicación que facilitaban la organización de pasantias prácticas y el desarrollo de los "saber hacer" y de las "destrezas" profesionales.

Por el contrario, los profesores de la enseñanza secundaria eran 
contratados exclusivamente después de haber ganado el concurso de la Agregación, preparado en la universidad $y$ que valida exclusivamente conocimientos "académicos" ligados a la asiganatura. Este modelo de formación, como lo describe J.P.Hédouin, jefe de la MAFPEN de la academia de Créteil, "Valoriza la formación intelectual y los estudios universitarios: es ante todo, gracias a un "Alto nivel" del dominio de conocimientos "Cientificos" que se puede uno convertir en docente, el resto es asunto de talento y hasta de carisma...".

Me atravería a decir que el compartir estas preocupaciones cayó progresivamente en desuso, después de la segunda guerra mundial por razones ligadas a ambos grados de enseñanza.

Inicialmente, el incremento del nivel general de las calificaciones que demandaba el desarrollo tecnólogico, generó progresivamente a una modificación profunda de los programas de la escuela primaria. Ya no se trataba de enseñar a leer, a escribir y durante tres años, mediante concurso, al finalizar el cuarto año de secundaria (noveno grado en Colombia) $y$

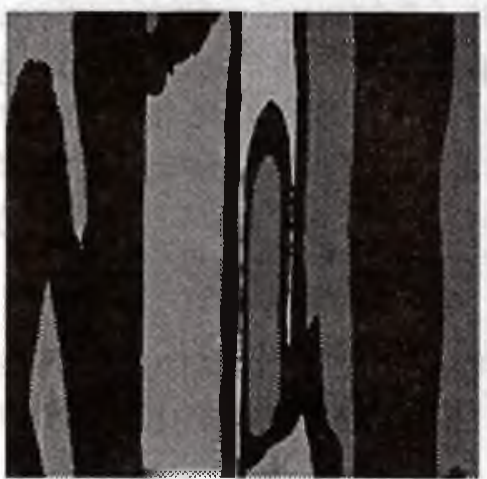

llerato, luego, a partir de 1969, son admi-tidos con el bachillerato y reciben dos años de formación profesional complementaria. Finalmente a partir de 1986, las escuelas normales admiten estudiantes que ya tienen el DEUG (Diploma universitario de estudios generales), es decir, dos años de estudios después del bachillerato y les dan dos años de formación profesional.

El acceso al estudio por parte de los jóvenes contemporáneos, nacidos inmediatamente después de la guerra, modificó, al mismo tiempo, la sociología del nivel de educación secundaria, que progresivamente ha dejado de ser un nivel de élite y se ha abierto a niños para los cuales dejó de ser operante una pedagogía de la limitación y del modelo familiar.

La creación de los Certificados de Aptitud para la Enseñanza Secundaria (CAPES), en 1950, fue seguida por la creación, en 1952 de los Centros Pedagógicos Regionales que organizaban algunas conferencias pedagógicas y pasantías en colegios y liceos (Básica Secundaria y Media en Colombia) con profesores "veteranos". A partir de 1980, los estudiantes que han tenido éxito en las pruebas teóricas del CAPES, son asignados a un grupo para el cual deben asegurar seis horas de enseñanza por semana, como titulares de una asignatura, mientras continúan recibiendo de formación pedagógica y didáctica complementaria. Es, casi en general, el sistema actual adoptado por los IUFM. 


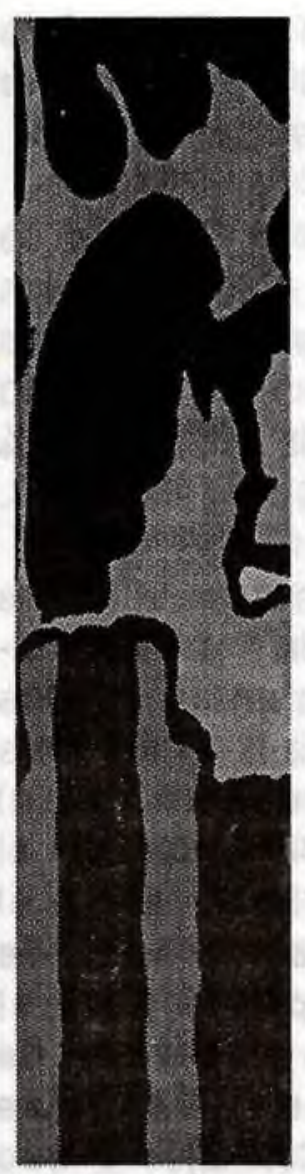

Estas instituciones tienen un volumen de actividad muy variable, según la extensión geográfica de la academia, su demografía, la concentración y la actividad de las universidades. Por ejemplo, la Academia de Córcega Los actuales IUFM, creados por la ley de Orientación sobre la Educación del 10 de julio de 1989, están a cargo de la formación inicial de los maestros de primero y segundo nivel.

Desde 1991, existen un IUFM en cada una de las 24 academias del territorio francés (tres en la región llede-France) y en los

departamentos y territorios de Ultramar (una academia corresponde, salvo por dos excepciones, a una región administrativa), podríamos comparar las academias con las Secretarías de Educación Departamentales o Municipales de Colombia, en algunas de sus funciones.

El número de inscritos se ha incrementado progresivamente desde el año de su creación (de 55.781 en 1991 - 1992 a 82.966 en 1994 - 1995), es decir un aumento del $49 \%$.

Esos 82.966 estudiantes se distribuyen asi: 56.450 en primer año y 26516 en segundo año, a los cuales se agregan profesores de primaria en formación específica.

albergaba en 1994 - 1995, 264 estudiantes, Limoges 589 , Versalles 5.270 y Lille 6.961 . El número de inscritos de una academia de tamaño mediano como París (5.996) en lo que se refiere a la enseñanza primaria y secundaria, se explica por la gran concentración de universidad en la capital.

La enseñanza es impartida en varios sitios, a menudo uno por departamento, instalado en las antiguas escuelas normales que formaban institutores (maestros de primaria).

Los IUFM tienen la categoría de establecimientos públicos de educación superior de carácter administrativo, están adscritos, por convención a una o varias universidades (ocho, por ejemplo, en el caso del IUFM de parís).

Los formadores son docentes investigadores de las universidades o docentes adscritos al Instituto (directores de estudios, antiguos profesores de escuelas normales) 0 inspectores de la Educación Nacional, para el primer nivel, o inspectores pedagógicos regionales para el segundo nivel, o maestros formadores para el primer nivel o para la enseñanza secundaria, profesores de colegio o de liceo descargados parcialmente de sus horas de cursos y escogidos por sus competencias en madera pedagógica y didáctica. 
El equipo de dirección está constituido por un director y directores adjuntos, en el mejor de los casos, el equipo incluye universitarios y miembros del cuerpo de inspectores.

EI IUFM es administrativo por un Consejo Administrativo, presidido por el rector de la academia.

inscritos desde 1971 ha sido la más espectacular: $116 \%$ para el primer grado, $92 \%$ para el segundo grado.

La preparación para el concurso de primer grado ocupa al $27 \%$, de los inscritos las diferentes preparaciones a los concursos de segundo grado ocupa el $72 \%$, de los cuales el $53 \%$ es para el CAPES.

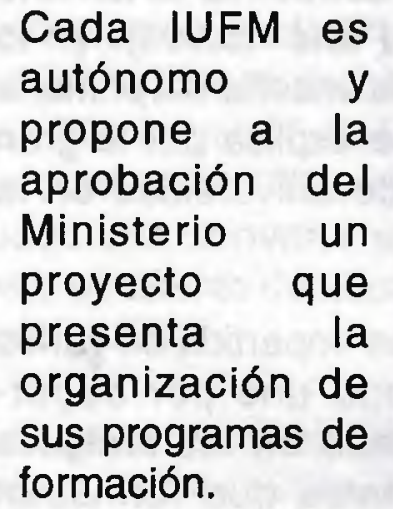

El nivel de admisión es idéntico para ios dos grados de enseñanza; la autorización para inscribirse está sujeta a la posesión de una Licenciatura, o de un diploma homologado como equivalente que certifique una formación de tres años después del Bachillerato.

\section{El Primer Año}

En todos los casos, en el primer año, los estudiantes del IUFM se consagra a la preparación de un concurso externo. Aquí la progresión de

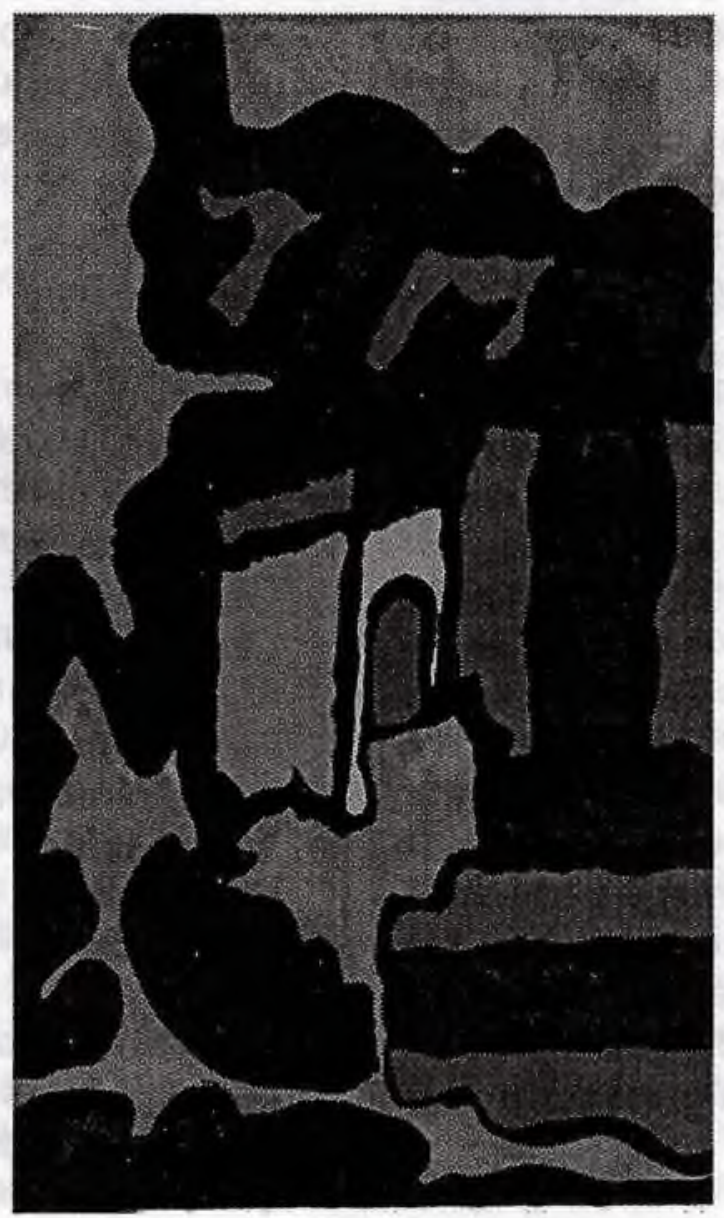

En lo que se refiere a la enseñanza elemental, los c a n d id a to s preparan, en el IUFM, durante el primer año, el Certificado de Aptitud para el Profesorado de las Escuelas (CAPE), que incluye pruebas para evaluar el dominio de las diferentes asignaturas que se enseñan en la escuela.

La admisión depende del dominio de la lengua francesa $y$ de las matemáticas; el examen de admisión comprende cuatro pruebas: una prueba escrita, para escoger entre historia - geografía, ciencias de la vida y de la tierra, ciencias físicas tecnología, una prueba oral para escoger entre las artes plásticas, la educación musical o una lengua extranjera, una prueba de educación 
física y deportes, una prueba sobre un trabajo documentado de contenido pedagógico y didáctico compuesta de una exposición oral seguida de una entrevista con el jurado.

Los aspectos de enseñanza incluyen por un lado una formación general y especifica, en proporción de $40 \%$ de formación general y $60 \%$ de formación en la asignatura, por otro lado seis a siete semanas de pasantía de práctica acompañada.

La principal dificultad pedagógica reside en la extrema variedad de las competencias y de los saberes, debido a una variedad, no menos extrema de licenciaturas concedidas por las universidades, en el marco de su autonomía.

Algunas academias con déficit de inscritos disponen de subsidios que permiten a los estudiantes ser remunerados durante su primer año (actualmente se asignan 60.000 Francos por año).

Según el informe entregado al Primer Ministerio por los inspetores generales Señores Borne y Laurent en 1992, según las estadísticas de los IUFM, del 40 al $57 \%$ de los estudiantes candidatos al concurso de admisión de profesores de escuela eran tituladas de una licenciatura que no estaba relacionada con la enseñanza en la escuela primaria (Sicología, Ciencias de la Educación, Derecho, etc.). He aquí un problema de heterogeneidad que no se ha resuelto hasta la fecha, dada la gran dificultad que haya para poner en marcha itinerarios de formación individualizados.
En lo que se refiere a la enseñanza secundaria, los estudiantes pueden preparar seis tipos de concursos diferentes:

- El Certificado de Aptitud para Profesores de Enseñanza Secundaria (CAPES), para las asignaturas literarias y cientificas.

- El Certificado de Aptitud para profesores de Enseñanza Técnica (CAPET), para las disciplinas tecnológicas.

- El Certificado de Aptitud para Profesores de Educación Física y Deportes (CAPEPS).

- El Concurso de Admisión para la función de Consejero Principal de Educación (CRCPE).

- El Certificado de Aptitud para profesores de Liceo Profesional de segundo grado (CAPLP2), para enseñar enP liceo Profesional.

\section{- La Agregación.}

Para la preparación de las pruebas teóricas del CAPES, no es obligatorio estar inscrito en el IUFM, ya que puede hacerse como candidato libre. Por el contrario, este concurso incluye, además de las pruebas relacionadas con los conocimientos "académicos", una prueba profesional llamada hoy prueba sobre análisis documental. Dicha prueba que prepara al futuro profesor para llevar a cabo una reflexión metódica sobre las prácticas en la enseñanza, se prepara generalmente en el IUFM. Las pruebas de contenido "académico" se siguen preparando en las 
universidades, en el marco de convenios firmados entre éstas y los IUFM.

El concurso de la Agregación se prepara siempre en la universidad. Después de ganar el concurso, los profesores agregados entran al IUFM para el segundo año de estudios en el cual reciben una formación profesional.

\section{EL SEGUNDO AÑO:}

En el segundo año, los estudiantes ya han ganado algunos de los concursos y reciben una formación de carácter más profesional; ya tienen el estatus de profesores en pasantía. En esta etapa son remunerados.

El segundo año esta constituido por una pasantía de responsabilidad, asegurando de cuatro a seis horas de clase por semana, la elaboración de módulos de enseñanza, y la redacción de un documento.

La pasantía tiene lugar, como su nombre indica, en un grupo del cual es responsable el aspirante y recibe la ayuda de un consejero pedagógico. Dicha disposición se aplica durante el primero y segundo grado para quienes han obtenido la "Agregación y el Certificado". Los estudiantes del segundo grado que realizan una pasantía pueden recibir un grupo de colegio (educación básica secundaría) o de liceo (educación media). En estas clases reciben la visita de formadores de IUFM con el objetivo de aconsejarlos y también para preparar la validación final del año.
Quienes están en pasantía en la enseñanza secundaria se benefician igualmente, en la mayoría de los casos, de otra pasantía llamada "de práctica acompañada", que se realiza en la clase de un profesor titular; esta segunda pasantía se desarrolla en el nivel del liceo para los aspirantes que tienen bajo su responsabilidad un grupo en colegio, y en colegio para aquellos que tinen a cargo un grupo de liceo.

Los aspirantes que hacen pasantías en disciplinas tecnológicas desarrollan por su parte una pasantía en empresas, con una duración de seis a doce semanas.

Las enseñanzas se distribuyen en módulos de formación general y módulos de formación disciplinaria, es decir ligadas a las disciplinas o asignaturas y a su enseñanza.

Las formaciones generales son a menudo comunes a los estudiantes que se preparan para la enseñanza de primer nivel y quienes se preparan para la enseñanza secundaria en las diferentes disciplinas.

La tipología de dichas formaciones es bastante diversa y varia, naturalmente, de un instituto a otro de un año a otro. Se podrian distinguir a nivel general, formaciones relacionadas con:

- El saber ser del profesor, requisitos previos si se puede decir, a su actividad profesional por ejemplo: dominio de la voz, de la articulación, del gesto. 
- El conocimiento del sistema educativo y de la vida escolar: la institución, el proyecto de establecimiento (Proyecto Educativo Institucional en Colombia), los procedimientos de orientación, los derechos y los deberes del profesor, sus responsabilidades, la prevención de accidentes, de epidemias, del consumo de drogas, etc.

- La sicología del alumno y la sociología de la educación: el alumno en dificultad, la enseñanza en zonas difíciles, la motivación de los alumnos, las relaciones con los padres, el conocimiento del ambiente, la formación de alumnos parcialmente francófonos...

- Los componentes de la actividad educativa: el refuerzo cognitivo, la evaluación, la definición de objetivos, la construcción de secuencias, el trabajo en grupo, el manejo de ayudas particulares como el audiovisual y la informática...

- La apertura a otros campos transdisciplinarios o periescolares: conocimiento de las artes, del patrimonio, de las otras culturas.

Las formaciones ligadas a la enseñanza de las disciplinas hace énfasis para el primer grado, en las diferentes disciplinas enseñadas. Para el segundo grado los aspirantes son generalmente repartidos en grupos, según pertenezcan a colegio 0 , a liceo. Se trabaja en prioridad sobre la práctica en los grupos y asignaturas que tienen a su cargo. Este es a menudo el deseo de los aspirantes.

Pero veamos que se han desarrollado también reflexiones "horizontales" por ejemplo: la integración de las actividades ligadas a la lengua, a la literatura y a la cultura en una enseñanza de la lengua materna $o$ extranjera, o reflexiones "verticales", "curriculares", por ejemplo sobre la lectura de obras integrales, o sobre el enfoque de las categorías gramaticales desde el grado sexto hasta el grado terminal, etc.

Quienes realizan la pasantía de primer grado y quienes ya han obtenido los certificados son sometidos al redacción de un "mémoire" o monografía, documento de unas treinta páginas, relacionado con una situación profesional debidamente analizada y clarificada por medio de reflexiones y aportes teóricos pertinentes. Se supone que esta monografía constituye una unión entre la práctica y la reflexión teórica. 
Al final del año la validación de los "certificados" incluye los tres elementos de la formación: enseñanza, monografía y pasantía; cada uno de estos elementos es evaluado por una comisión de especialistas. Si las tres evaluaciones son positivas, el director del instituto propone la validación del año a un jurado académico, compuesto en su mayoría por miembros externos al IUFM. El año pasantía de los profesores que tienen la agregación es validado por medio de una simple inspección.

\section{Dificultades, avances, perspectivas.}

1. Unos de los objetivos anunciados cuando se crearon lo IUFM era el de una cultura profesional común a las diferentes categorias de profesores, independientemente de nivel en que ejercían y de la disciplina enseñada.

Podemos decir que este objetivo sólo se alcanzo parcialmente, por razones a la vez conyunturales y fundamentales.

Las razones coyunturales son múltiples, ligadas esencialmente al estatus y a la cultura corporativa de las diferentes categorías del personal involucrado:

- El personal de las antiguas escuelas normales, promovidos como maestros asistentes o maestros de conferencias en los institutos recientemente creados, quienes habían adquirido una sólida experiencia de la enseñanza primaria y de los problemas que allí se presentan. Por el contrario, aún si un buen número de ellos provenían de allí, ya habían perdido el contacto con la enseñanza secundaria en ese momento o paralelamente a la creación de los IUFM. Varias transformaciones importantes estaban ocurriendo (reorganización de la enseñanza general en tres grandes secciones: científica, literaria, económica y social, sistematización de la evaluación de los alumnos, a la entrada en sexto grado, a la entrada en segudo (décimo grado en Colombia) introducción al liceo de módulos de enseñanza centrados en las necesidades individuales de los alumnos, etc).

Muchos de estos formadores, por falta de legitimidad en lo que se refiere a la enseñanza secundaria, se refugiaron entonces en una enseñanza de la didáctica específica para la educación primaria o en módulos sicopedagógicos generales a menudo muy teóricos. Un ejemplo significativo es el de los profesores de filosofía a los cuales se les había confiado, en las escuelas normales la enseñanza de la sicología del niño y de adolescente, mientras que se le había alejado

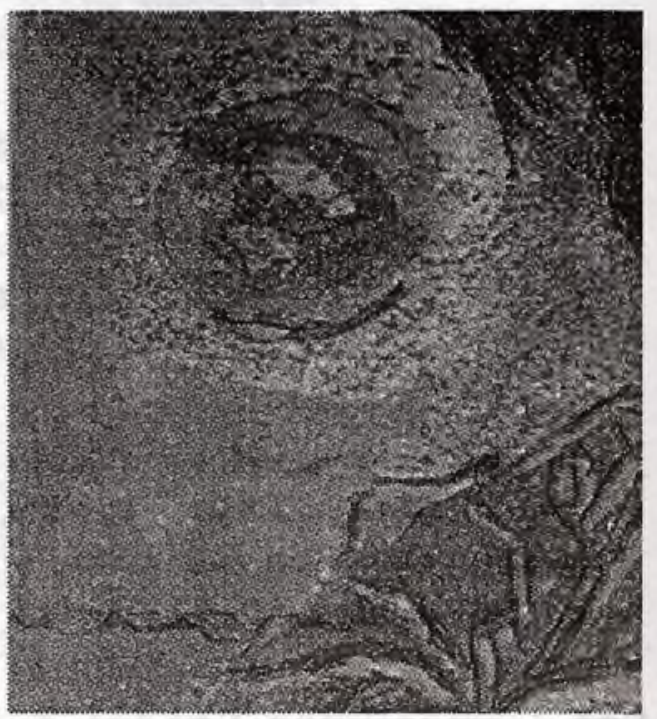




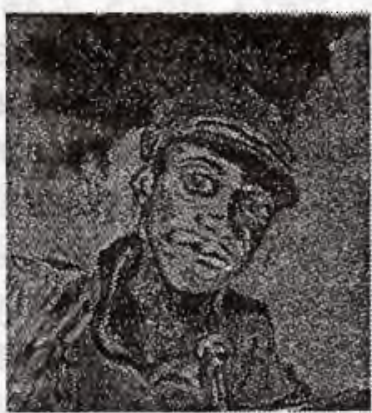

progresivamente de una reflexión sobre la didáctica de la filosofía, se les pedía de pronto manifestar en este campo una capacidad que no poseían.

- El personal adscrito a los antiguos C.P.R. ; por el contrario, estaba constituido en su mayor parte por delegados quienes aseguraban de acuerdo a la demanda y bajo la tutela de un grupo de inspección, seis horas de formación didáctica para los profesores de colegio y de liceo. Se le pordría reprochar sin duda a algunos de ellos la focalización un poco excesiva sobre los ejercicios más "reglamentarios" usados en la enseñanza de secundaria, ligados en particular en la preparación de exámenes, el Diploma Nacional, al finalizar el colegio y el Diploma del Bachillerato al finalizar el liceo. Pero la mayor parte de ellos había desarrollado, en el marco de los C.P.R., una considerable cultura didáctica, asociando el conocimiento y la enseñanza concreta del terreno a una investigación más distanciada sobre los objetivos y los contenidos de su disciplina; por el contrario la enseñanza elemental era para ellos un campo mal conocido, en el cual muy poco de ellos tenían el deseo de aventurarse. $Y$ aunque lo hubieran deseado, faltaba todavía facilitarles el acercamiento gracias a una formación de formadores, que en la mayoría de los IUFM, aún no se ha construido.

- Los universitarios, una parte de ellos rechazó la creación de nuevos institutos, la otra parte - no siempre la más calificada - vio allí la ocasión de hacer carrera en el campo de la didáctica, menos complicado que el de la lingüistica o de la historia literaria, aportando puntos de vista teóricos a veces apoyados sobre un conocimiento antiguo, y hasta de segunda mano, de la realidad de la enseñanza.

2. Pero más allá de estas dificultades conyunturales, más o menos especíticas de la realidad francesa, que menciono aquí con la intención de evitar a otros el caer en problemas ánalogos, se presentaban y se presentan todavía un cierto número de problemas fundamentales, que tratan de encontrar soluciones aquí o allá y que abren perspectivas de progreso y de desarrollo.

- Los estudiantes entran al IUFM con un pasado escolar y universitario muy diverso, debido en particular para el primer grado a la variedad de las licenciaturas admitidas para postular al concurso de las escuelas, y para el segundo grado, a la diversidad de los contenidos de las formaciones universitarias, por causa de la variedad y para decirlo todo, de la calidad desigual de las unidades de valor que componen los diplomas anteriores, el diploma universitario de estudios generales (DEUG) y las licenciaturas en las diferentes disciplinas. 
- Más profundamente el perfil sicológico, las motivaciones, los proyectos, la concepción que se hacen los estudiantes de su futura carrera delimita itinerarios individuales de extrema diversidad: someramente podemos ya distinguir dos grandes familias: la de aquellos que aman una disciplina y para quienes la enseñanza es el único sustento que les permite vivir en contacto, deprimente a veces, con esta disciplina. A menudo se les encuentra, claro esta, entre los estudiantes que preparan un concurso para la enseñanza secundaria. Aquellos que tienen a menudo una representación de su oficio muy alejada de la realidad de los liceos y sobretodo de los colegios. Ellos tienen en mente un modelo de enseñanza muy cercano a la universidad y viven a menudo su situación de profesores bajo el modo del "último recurso" y de la frustración. Su formación debería consistir esencialmente en señalar la unión estrecha indisoluble, entre la constitución de los saberes y su eficacia didáctica, en trabajar los lazos múltiples y recíprocos entre los conocimientos y los métodos específicos de cada disciplina, por una parte, y por otra parte lo que hemos convenido en llamar hoy la educabilidad congnitiva. EI aprendizaje forma el espíritu como el espíritu produce conocimientos.

La otra familia está conformada por aquellos que han escogido el oficio de mestros por afición a los niños, por el gusto a la animación, por militancia pedagógica, por confianza en el ser humano. Escoger el campo de la enseñanza es para ellos casi secundario. Este perfil se encuentra evidentemente, sobre todo entre los futuros profesores de la educación primaria, la cual es por naturaleza, pluridisciplinaria. Pero también se encuentra entre los profesores y futuros profesores de sucundaria quienes durante su carrera universitaria han dudado en escoger entre las letras, las matemáticas la historia y la física. Sobre este tema se han construido las campañas de admisión a los IUFM con slogans como "Profesión profesor".

A esto hay que convecerlos de que, por más indispensables que sean, las cualidades de animación apenas constituyen una parte de las competencias del profesor y que la materia enseñada juega en la formación un papel tan importante como el proceso de construcción de conocimientos. No hay forma sin materia informe.

Agreguemos sin vergüenza que la situacuón actual, del mercado de trabajo atrae más hacia los cargos administrativos, especialmente en las áreas de la educación, un número creciente de jóvenes que ocupanuna posición intermedia en este esquema.

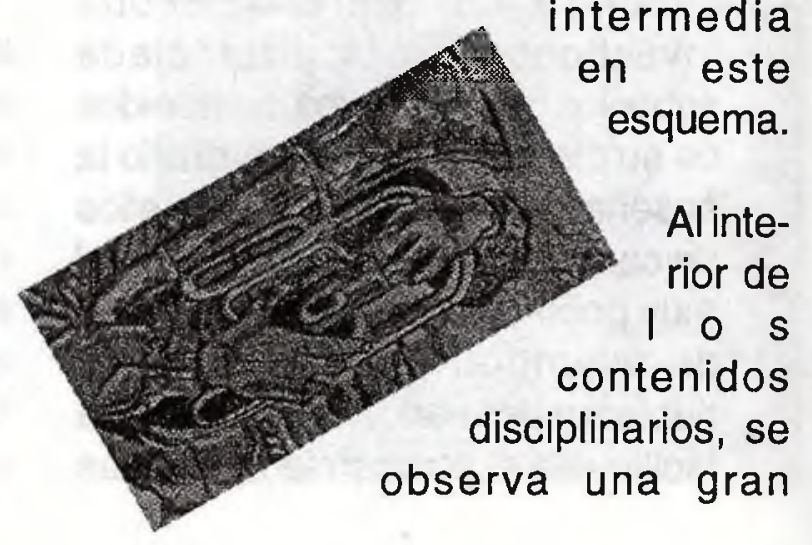


variedad de saberes, especialmente en la distribución entre los diferentes sub-áreas que componen una disciplina. Muy a menudo en el ejercicio de la profesión el desvío didáctico operacional pasa por una "integración" entre las sub-áreas de la disciplina; para dar dos ejeplos rápidos ocurre a menudo en matemáticas, que una particularidad algebraica puede

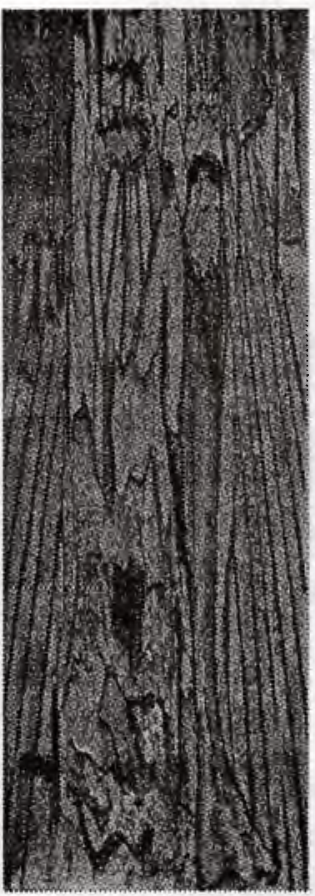
demostrarse por una construcción geométrica (por ejemplo la identidad sobresaliente: $(a+b) 2=$ $(a 2+2 a b+b 2) d e$ la misma manera, cualquier enfoque de textos literarios o no, supone la movilización conjunta de saberes relativos al léxico, a la organización de los textos, a los referentes culturales asociados.

Esta integración supone por su parte del profesor un dominio semejante de las diferentes sub-áreas de su disciplina, lo que está lejos de ser el caso general, tanto al iniciarse en los IUFM como en el transcurso de la carrera, -y lo digo con conocimiento de causa-.

Se trata entonces de llevar este público tan heterogéneo al nivel de conocimientos académicos y de competencias profesionales requeridos para ejercer el oficio de profesor.

Hoy en día la reflexión de la mayoría de los institudos se centra en los problemas surgidos del manejo pedagógico de la heterogeneidad, en el marco de la elaboración de sus proyectos plurianuales.

Tanto en materia de formación de profesores como en materia de educación de los alumnos, la capacidad para manejar la heterogeneidad supone la elaboración de tres tipos de formación:

- Un "inventario" o referencial de las competencias profesionales del profesor en materia pedagógica y didáctica.

- Procesos de evaluación y de ubicación con respecto a dicho referencial.

- Modalidades individualizadas de tratamiento pedagógico o de referencial.

- Modalidades individualizadas de tratamiento pedagógico o de refuerzo.

Hay aquí una amplia perspectiva de desarrollo cualitativo que se abre apenas hoy y que dará, en el futuro, la medida del éxito de los IUFM.

La condición de dicho desarrollo está, evidentemente, ligada a la existencia de un dispositivo permanente de reflexión y de formación de formadores, que apenas se encuentra en la etapa de diseño.

Otro problema esencial que queda por resolver, es el Ide la validación 
equilibrada de la formación. El principio de base es que los tres elementos de la formación es decir, la enseñanza (general y didáctica), la monografía, el dominio de las prácticas de clase, tal como se manifiesta durante la pasantía de responsabilidad son evaluadas en partes iguales.

De hecho, si la práctica de la clase puede ser, $y$ de hecho es, objeto de una evaluación criterial, la formación recibida sólo podría evaluarse de manera relativamente objetiva por medio de controles finales 0 durante la formación. Pero la gran variedad de los módulos de formación propuestos y la variedad en las prácticas de los formadores dificultan una evaluación verdaderamente equitativa y objetiva. En consecuencia, los IUFM se limitan a menudo a evaluar la presencia de los estudiantes en las sesiones de enseñanza.

La monografía presenta un problema simple y complejo a la vez. Si debe ser verdaderamente una reflexión basada estrechamente en la observación y el análisis surgida en la práctica de clase, la monografía complementaría felizmente la evaluación de dicha práctica midiendo la capacidad del profesor para apreciar crítica y objetivamente la práctica y para relacionar la vivencia de la enseñanza y el saber teórico; pero todavía vemos aquí y allá monografías que están desconectadas de las situaciones vividas y se asemejan a pequeñas tesis de didáctica 0 de ciencias de la educación. Sería absurdo en este caso, rechazar la validación a estudiantes que tienen éxito en las prácticas con su grupo pero que no logran pasar este ejercicio un poco formal y poco útil.

Aún queda un camino por recorrer para que este dispositivo relativamente reciente este a la altura de su eficacia. Podría decirse desde ahora de después de las turbulencias bastante ideológicas que provocaron al ponerse en marcha, los IUFM funcionan de manera relativamente satisfactoria. A la espera de una evaluación cualitativa más rigurosa, yo como practico, observo características alentadoras entre los jóvenes 
profesores egresados de los IUFM, que transforman poco a poco las representaciones del oficio de profesor. En primer lugar, se observa una concepción menos solitaria del oficio, una mayor facilidad para trabajar en común, para intercambiar procedimientos $y$ herramientas

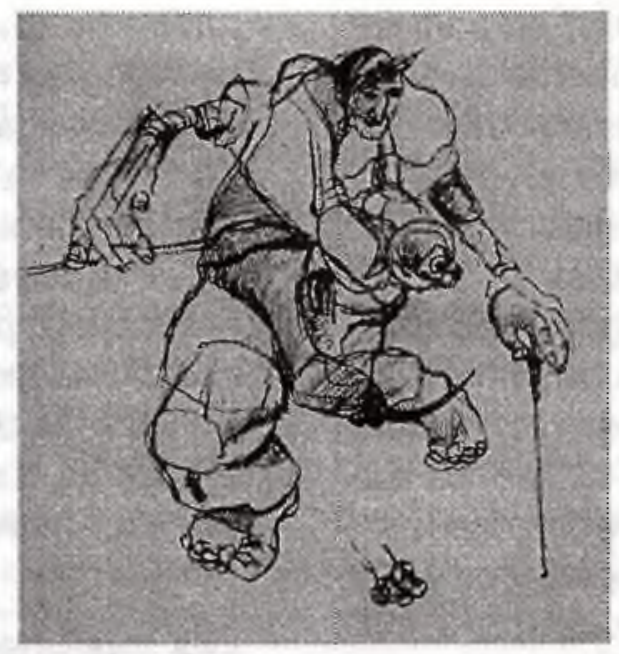

pedagógicas, para confrontar estrategias de aprendizaje, para participar activamente en los proyectos educativos institucionales.

Me parece que se ve aquí, el surgimiento de una verdadera mutación en la cultura profesional de los docentes cuyo mérito debe atribuirse sin duda a la formación impartida en los IUFM.

Pero dicha mutación sólo tendrá un efecto completo cuando la formación inicial sea retomada por un dispositivo de formación continua a lo largo de la carrera del profesor. La aplicación de dicho dispositivode formación continua a la enseñanza secundaria, es la segunda etapa de la reforma emprendida en los años 80 .

\section{LA FORMACIÓN CONTINUA}

La enseñanza Primaria, desarrolló especialmente, a nivel del departamento y de la región, programas anuales de formación, en los cuales los maestros formadores y los inspectores intervienen principalmente como formadores; este dispositivo presenta la ventaja de estar más próximo de los usuarios; presenta también el inconveniente de que dispone de una reserva reducida de formadores, en consecuencia, de una oferta de formación relativamente pobre que deja poca iniciativa a los institutores y constituye un mal relevo de políticas nacionale. Una de las razones por las cuales una reforma tan amplia y ambiciosa como la nueva organización en ciclos de la escuela elemental, basada en referentes que permiten obtener en todo momento una ubicación precisa de las competencias de los alumnos, transformó muy poco el comportamiento de maestros e instituciones. Las pasantías de sensibilización propuestas aquí y allá no llegaron a remplazar los seminarios tradicionales de iniciación a las artes plásticas o al vídeo, presentados año tras año.

En cuanto a la enseñanza secundaria, por el contrario, se trató no de una adaptación sino de una verdadera creación en la medida en que, hasta 1982, no existía ningún dispositivo de formación continua que fuera efectivo, como lo había para la formación inicial.

En efecto, mientras que en Francia se había desarrollado, desde 1971 , importante dispositivo de formación permanente de asalariados, para lograr la adaptación de los trabajadores a las evoluciones y 
mutaciones tecnológicas, permitir la reinserción de los solicitantes de empleo, jóvenes y menos jóvenes, facilitar el acceso de los jóvenes al mercado de trabajo e incrementar la cualificación de los trabajadores de bajo nivel, no existía ningún dispositivo comparable en materia de formación continua de los docentes, a excepción de los institutores que asistían a las "conferencias pedagógicas" organizadas por los inspectores y los seminarios de formación para los auxiliares

No nos tendremos sobre las razones, frecuentemente analizadas de este retraso: resistencia al cambio de un "medio tradicionalmente humanista" para mantener el sistema en "una oscuridad más o menos confortable pero organizado y familiar", como lo escribe M. Jobert, y concepción fija del saber, causando una evolución relativamente lenta de los contenidos y los métodos de enseñanza.

De hecho, cuando una disciplina como las matemáticas transformó desde los años 70 métodos y contenidos introduciendo las matemáticas llamadas "modernas", esta transformación, para decirlo de alguna manera, generó un dispositivos, instituido en 1974: los Institutos de Investigación sobre la enseñanza de las Matemáticas (IREM), verdadera prefiguración de las actuales estructuras de formación continua.

Pero en los años 80 aparece cada vez más claramente la necesidad de concebir un dispositivo de formación continua para los profesores, a la vez para ayudarlos a adaptar su pedagogía y métodos didácticos a las características de los nuevos perfiles de los alumnos, originados principalmente por la democratización y el acceso a la enseñanza secundaria, para tener en cuenta, en el caso de los IREM, la evolución de los saberes disciplinarios y para abrir la escuela al mundo exterior y en particular al mundo del trabajo.

Las misiones Académicas para la Formación Continua del Personal de la Educación Nacional (MAFPEN) fueron creadas pro el decreto del 12 de junio de 1982 , reemplazado por un nuevo decreto fechado el 25 de julio de 1985 . Sus tareas son definidas en la circular del 24 de mayo de 1982:

- Censo y análisis de las necesidades de formación mediante una consulta profunda de todo el personal.

- Inventario y análisis de los recursos y posibilidades de la academia.

- Elaboración de un plan académico coherente que responda a los objetivos generales de formación ligados a la renovación del sistema educativo así como a la demanda de educación permanente del personal.

- Elaboración progresiba de métodos de evaluación de las acciones realizadas. Estos métodos están dirigidos por un jefe de misión bajo la autoridad del rector, canciller de las univeridades. 
La actas de su seminario nacional de jefes de MAFPEN que se realizó en Lyon en enero de 1990, propone resaltar en la historia reciente de estas misiones, tres períodos:

Un primer período (1982-1984), caracterizado por un doble trabajo de inventario de la demanda individual de formación y constitución de una red de formadores. Los grupos de trabojo constituidos por disciplinas analizan las propuestas de seminarios y seleccionan los candidatos esencialmente en función de las habilidades o competencias de los formadores.

Durante este período se constituyó una red de formadores y se elaboró una metodología de diseño del Plan Académico de Formación (PAF), de acuerdo a un calendario muy riguroso: Identificación de necesidades, selección de las acciones propuestas en la apertura, publicación del catálogo para el año siguiente. Al inicio del año escolar: acopio y análisis de candidaturas y programación de acciones, lugares, fechas y respuestas individuales a los candidatos y formadores. Esta metodología de elaboración del PAF perdura todavía. Al contratio, esta primera organización limitada de alguna manera, a la oferta de formación y a la demanda potencial, tenía el inconveniente de permitir erróneamente la difusión de la innovación debido a "la dificultad, para el participante aislado, de integrar a su práctica los contenidos de formación recibidos y compartidos con sus colegas que no vivienron la misma experiencia". Esta formación tiene sobretodo como consecuencia perversa la de instaurar una "lógica de oferta", en virtud del peso de los formadores en los grupos de trabajo y de la tendencia muy conocida de los formadores a identificar como "necesidad" de formación aquello a lo que son capaces de adoptar una respuesta.

Un segundo período (1984-1988) contemporáneo de las leyes de descentralización, de la renovación de los colegios y de la promoción de proyectos educativos institucionales se caracteriza por una nueva estrategia de los MAFPEN, que se fundamenta en cinco preocupaciones:

- Analizar, descifrar en una situación determinada de trabajo, la demanda de formación que se expresa.

- Elaborar una respuesta a partir de las necesidades inventariadas y proponer formaciones "a la medida".

- Conducir y administrar la formación al nivel más local posible, es decir en al institución o con la integración de las instituciones de una misma zona geográfica.

- Reducir el plazo entre la formulación de la demanda de formación y la respuesta.

- Reducir los riesgos de ruptura entre las necesidades reales y las respuestas aportadas.

Los efectos de esta nueva estrategia son, por supuesto, extremadamente variables, en la medida en que su éxito está condicionado a la capacidad de la institución para analizar, formular, administrar sus necesidades de 
formación, (a manera de ejemplo, cualquier director de colegio definirá como prioritaria la demanda que transformará lo menos posible su horario) y por la capacidad de los equipos formadores para analizar las situaciones y definir las necesidades. Estos equipos se ven, desde entonces "obligados a veces, a ofrecer formaciones sobre temas generales de la renovación, sin estar siempre en condiciones de inscribir sus intervenciones en un proceso real de renovación. De aní que se corra el mismo riesgo que en la estrategia de formación anterior: dar una formación cuyo único fin es ella misma, sin poder medir el impacto de la formación sobre los cambios en curso".

Un tercer período (desde 1988) donde las misiones agregan a su actividad de form a c i ó $n$ propiamente dicha la misión de facilitar la negociación entre los diferentes niveles, formadores, personal o formar, instituciones en particular, interviniendo en calidad de asesores en al concepción de los proyectos educativos institucionles.

Estas evoluciones sucesivas conducen actualmente a planes de formación que diferencian en general varios tipos de acciones:
- Acciones abiertas para públicos "seleccionados" cuya formación corresponde a objetivos nacionales o académicos para grupos de personal particulares; por ejemplo: entre 1989 y 1993, ante la necesidad de preparar a los profesores para poner en marcha, en las mejores condiciones posibles, el dispositivo de evaluación de los alumnos de grado 6ㅇ, algunas academias elaboraron un dispositivo que permitió a todos los profesores que enseñan francés en este grado, recibir una formación específica por períodos sucesivos. Otro ejemplo: ante la oportunidad de una modificación profunda como la que afectó el año pasado las pruebas de estado en el área de francés, todas las a c a d e m i a s organizaron, cada una a su manera, cierto número de reuniones que permitieron informar a todos los profesores de francés del nivel de Educación media, liceos, los cambios en curso, e invitarlos a reflexionar sobre la incidencia de dichos cambios en sus prácticas.

Acciones de candidatura individual, cuyo tema corresponde, obviamente, a prioridades académicas, pero a las cuales los profesores de la materia y del nivel 
involucrados, eligen inscribirse en función de sus intereses y necesidades personales.

Acciones para responde a la demanda de grupos Institucionales, que buscan acompañar un aspecto particular del proyecto de la institución; estas acciones no aparecen en el catálogo de cursos ofrecidos por el PAF, pero son negociadas con los grupos que las socilitan.

A esto se agregan diversas actividades particulares, como la actualización de los profesores que hayan sufrido interrupciones 0 accidentes durante su carrera, a causa, especialmente de enfemedad, preparación de concursos internos, organización de pasantías prácticas de los profesores adminitos por concurso interno, etc.

A la acción de las MAFPEN se agregan, a nivel nacional, dos clases de dispositivos: un Programa Nacional de Formación (PNF), que corresponde a las prioridades definidad por la Dirección del Ministerio (Escuelas, Liceos y Colegios, personal administrativo; un grupo de universidades de verano, propuestas por instituciones, formadores, movimentos pedagógicos, con la aprobación del Ministerio). Estas universidades, cuyo catálogo aparece en el Boletín Oficial de la Educación Nacional, funcionan durante las vacaciones escolares $y$ reúnen profesores voluntarios que asisten a la formación durante su tiempo de vacaciones y participan en la financiación de dichas acciones de formación.
Dificultades, avances, perspectivas

Este conjunto considerable de actividades, tiene, evidentemente, un efecto importante en al evolución de las competencias profesionales de los profesores. El sistema puede sin duda, ser mejorado. El peso de la oferta de formación sigue siendo considerable con respecto a las necesidades que pueda ser identificadas por los mismos profesores, por los jefes de establecimientos y por los funcionarios de inspección.

Todavía es bastante difícil manejar la renovación de los formadores. Esta renovación es sin embargo necesaria, por razones evidentes: si los formadores son contratados en función de competencias precisas que responden a necesidades hagan surgir la demanda de otros formadores, dichos formadores demasiado inveterados, tienden inevitablemente a constituir grupos de presión que pretenden someter la oferta en el sentido que es favorable a su perpetuación; la profesionalización de los formadores no es deseable, en cualquier caso, por razones de eficacia y legitimidad

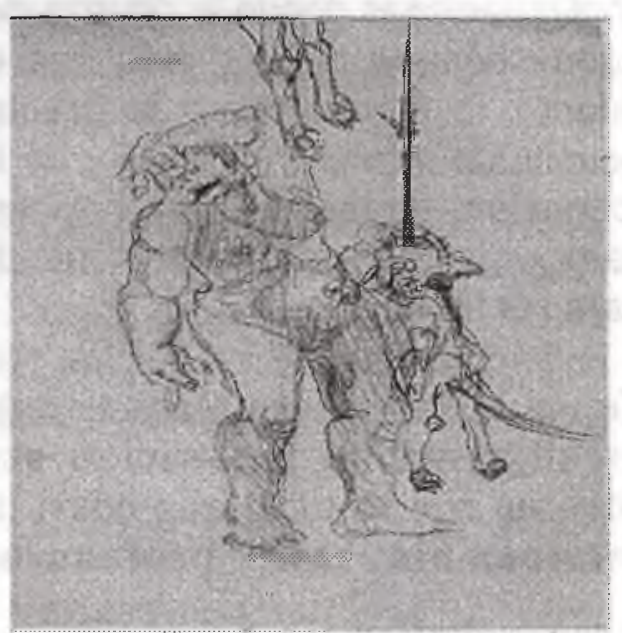


pedagógica. Los formadores son en su mayoria, profesores que han desarrollado, en su práctica de clase y gracias a una reflexión y a una teorización particular, competencias uqe hacen transmisible su excelencia pedagógica. Este tipo de competencias que se fundamenta a la vez en saberes y en prácticas, envejece mal por fuera del contacto con la realidad escolar, pero en el imaginario colectivo, es más honorable ser formador que profesor raso y volver a ejercer el oficio es visto como una regresión, un retroceso y hasta una desaprobación por parte de la institución.

Por ello es tan delicado despedir a un formador, aunque se tenga a veces mucha urgencia de hacerlo.

Además el talento que se requiere de un formador, no es siempre, como cada uno sabe, el reflejo fiel de sus competencias técnicas. En nuestra cultura, aún entre profesionales excelentes, la formación esta siempre más o menos representada de un modo carismático, sin duda porque esta concebida esencialmente como una transmisión de conocimientos según un modelo universitario. Otras configuraciones donde la riqueza del aporte pesaría tanto y más que el talento, la elocuencia y la gracia, permitirían movilizar alrededor de la formación, profesores que hoy nos parece que no tiene el perfil requerido para ser formadores.

Ya que las formaciones más prestigiosas de innovación son aquellas que se organizan como un intercambio entre profesores comprometidos en un mismo proceso de renovación y de investigación, lo ideal sería poder asegurar el seguimiento de las fórmaciones por medio de redes de información y de intercambios que permitan la comunicación de herramientas y procedimientos pedagógicos. Es necesario orientarse hacia este cuarto tipo de dispositivo; hoy en día las dificultades son numerosas. Una de ellas, y no de las menores, en la de que actualmente no se ha previsto el remplazo en el establecimiento, para los profesores en formación. Esto origina la reticencia de los directores y por consiguiente, es una de las razones por las cuales no se le concede la suficiente importancia a la formación de los proyectos educativos institucionales. Se deben imaginar soluciones como el remplazo de profesores que estén en programas de formación investigación por profesores titulares por supernumerarios o por practicantes en formación para quienes este remplazo constituiría un elemento de la formación profesional.

Finalmente es necesario que los formadores puedan confrontar sistemáticamente sus teorías y sus prácticas al interior de un dispositivo permanente de concertación y de formación de formadores, lo cuál está todavía muy lejos de ser el caso, ya que cada uno prefiere, a menudo, cultivar todavía ilusoriamente su especialidad. \&

Traducido al español por: Lucía Elena ESTRADA MESA 